IP-ASTP-02-94

April, 1995

\title{
Eigenstates of the Vorticity Operator: The Creation and Annihilation of Vortex States in Two Dimensions
}

\author{
A. D. Speliotopoulos \\ Institute of Physics, Academia Sinica \\ Taipei, Taiwan 115, R.O.C.
}

\begin{abstract}
A quantum mechanical description of vortices in two dimensional superfluid ${ }^{4} \mathrm{He}$ flims is presented. Single vortex creation and annihilation operators are defined and wavefunctions for these states are explicitly constructed. A hamitonian for these states is then proposed.
\end{abstract}

PACS Nos.: 67.40.Vs, 67.40.Db, 03.65.Bz. 


\section{$\S 1$. Introduction}

As is well known by now, the phase transition of the two dimensional superfluid ${ }^{4} \mathrm{He}$ film is quite well described by the Kosterlitz-Thouless (KT) phase transition [1]- [4] which makes explicit use of the presence of a gas of point vortices in the superfluid. As the dynamical properties of these vortices must be known before the critical properties of the phase transition can be calculated, an analogy is made between the superfluid and the classical ideal liquid. Kirchoff's equation of vortex motion can then used to define a grand canonical ensemble for the system and the critical indices can be calculated. Superfluidity, however, is believed to be a quantum mechanical phenomenon while KT phase transition relies on classical fluid dynamics [5] to construct a classical grand canonical ensemble. The only place quantum mechanics is used is in the requirement that the "charge" or vorticity of these vortices must be integral multiples of $h / m$. It is therefore quite natural to wonder if a quantum mechanical description of these vortices is possible.

The purpose of this paper, therefore, is to develope a quantum mechanical description of vortices in superfluid ${ }^{4} \mathrm{He}$ films. There are two approaches that one can take. The most straightforward, naive approach would be to continue treating the vortices as point particles in and of themselves and attempt to "quantize" their classical equations of motion directly. When one tries to do so, however, one is immediately presented with seemingly insurmountable problems caused, ironically enough, by the very simplicity of Kirchoff's equations themselves. If we label the coordinate of a vortex by $(x, y)$, then from Kirchoff's equations the momentum conjugated to $x$ is the coordinate $y$. As Onsager [7] first noted, naive canonical quantization would then seem to give the non-sensical result that the two coordinates do not commute: $[x, y] \neq 0$. Moreover, because the classical equations of motion depend only on the velocity of the vortices, their lagrangian is linear in their velocities. Consequently, their classical hamiltonian does not contain a kinetic piece, but is instead all potential. One does not have a "free" hamiltonian which can be perturbed about and must instead deal immediately with the fully interacting theory. Nevertheless, this approach has been attempted by McCauley [8] who implimented the canonical quantization condition by everywhere replacing, by hand, the coordinates $x$ and $y$ of the vortices with the raising and lowering operators of the SHO. Although certain results, such as the finite core size of 
the vortex, can be obtained in this manner, this replacement is ad hoc and only a system containing at most two vortices with fixed circulations can easily be treated by it. The total vorticity is necessarily fixed in this approach. In addition, it is not altogether certain that the two dimensional vortex gas can be consistently quantized in this manner, as has been argued in [9], [10].

The approach we shall follow in this paper is based on the observation that we already know which states of the fluid are important and necessary to describe the superfluid phase transition. Namely, they are states of definite vorticity. With the states of the fluid known, we then need only find the relevant physical operators for these states. By considering the vorticity as a good quantum number for the system we can define a vorticity operator $(h / m) \mathcal{Q}$ for which these states are eigenstates of. The eigenvalues of $\mathcal{Q}$ shall then simply be the vorticity of the system in units of $h / m$. Using complex coordinates, it is straightforward to develope a heuristic derivation of these states and, through them, a coordinate representation of $\mathcal{Q}$. With this in hand, we shall, as in the case of the simple harmonic (SHO), be able to define ladder operators, creation and annihilation operators, $c$ and $c^{\dagger}$, whose operation on eigenstates of $\mathcal{Q}$ takes one from one state of definite vorticity to another. A hamiltonian $H_{c} \sim c^{\dagger} c$ can then be defined, which, importantly, commutes with $\mathcal{Q}$. States of definite vorticity in the fluid may then be eigenstates of both $H_{c}$ and $\mathcal{Q}$. Moreover, the algebra of operators closes, and no other operators need to be introduced. As the wavefunctions and the relevant operators are known, the quantization of the single vortex system is then complete.

This approach is the very reverse of the proceedure one usually follows when quantizing a classical theory. One usually starts with a hamiltonian and construct from it the wavefunctions and Hilbert space. In our case, we know what the relevant states of the system are, and from these states we shall construct the hamiltonian and the other relevant physical operators of the theory. This approach has obvious draw backs, of course, not the least of which will be a hamiltonian which is significantly different from the one one usually encounters. We can only be certain that this hamiltonian is the relevant one by showing that it, and its eigenvalues, has many of the same properties as the classical hamiltonian. It is also only in this way that the unknown constants in the theory can be fixed. 
The rest of this paper is organized in the following manner. $\S 2$ is devoted to reviewing certain properties of classical vortices, in particular the difficulties inherent in the simplistic canonical quantization of the vortex gas. In $\S \mathbf{3}$ we shall define and construct the algebra of operators which are relevant to the description of a single point vortex in the superfluid. Following the analysis of the SHO, the single vortex Hilbert space shall be constructed. This construction is purely algebraic, however, and we shall delay showing that a faithful representation of the algebra can be chosen until $\S 4$ where the single vortex wavefunctions will be constructed and interpreted. Concluding remarks can be found in $\S \mathbf{7}$.

A brief comment on terminology. Because vortices in quantum liquids have "quantized" circulation, one often refers to these vortices as "quantum vortices". This is to differentiate these vortices from vortices found in classical fluids whose circulations need not be integer valued. This terminology may, however, be confusing and misleading in the current context since in no way are the motion of vortices quantized. Because of its usefulness, however, and its prevelance in the literature, we also shall use it. Nevertheless, we caution the reader against any possible misunderstanding which may result.

\section{$\S 2$. Background}

We begin with a brief review of the standard theory and application of quantum vortices to superfluidity. This review is necessarily cursury and the reader is refered to [11] for further details. Although we are working with vortices in two dimensional films, we shall begin by considering vortex lines in three dimensions. This is partly because the traditional argument for the presence of point vortices in two dimensional films follows in direct analogy to the three dimensional case. Mainly, however, it is because certain problems and inconsistencies araises in this argument when one actually tries to impliment it in two dimensions [12]- [14]. We shall delay discussion of such problems until later in the section, however, and shall gloss over for now any problems which may araise.

We start with the standard definition of what is meant by a quantum vortex in three dimensional ${ }^{4} \mathrm{He}$ superfluids. Let $\psi$ be the microscopic bosonic field for ${ }^{4} \mathrm{He}$ and consider its thermodynamic average $\langle\psi\rangle$. In the normal fluid phase this average vanishes due to the $U(1)$ gauge invariance of the hamiltonian for the theory. In the superfluid phase, on the other hand, $\langle\psi\rangle \neq 0$, denoting the breaking of the $U(1)$ gauge symmetry. A current density 
for $\langle\psi\rangle$ can then be constructed as

$$
\vec{j}=\frac{\hbar}{2 m i}\left(\left\langle\psi^{\dagger}\right\rangle \vec{\nabla}\langle\psi\rangle-\vec{\nabla}\left\langle\psi^{\dagger}\right\rangle\langle\psi\rangle\right)
$$

where $m$ is the mass of the boson. If we then polar decompose $\langle\psi\rangle=\rho_{s}^{1 / 2} \exp (i \alpha)$,

$$
\vec{j}=\frac{\hbar \rho_{s}}{m} \vec{\nabla} \alpha
$$

Then, because the microscopic field is a boson,

$$
\oint_{\gamma} \frac{\vec{j}}{\rho} \cdot d \vec{l}=2 \pi q \frac{\hbar}{m},
$$

where $\gamma$ is any closed path in the fluid and $q$ is an integer. From analogy with fluid dynamics, eq. (3) is called the circulation of a vortex. Because $\vec{j}$ is proportional to a total derivative, the circulation is independent of the shape $\gamma$. Since $q$ is an integer, at this point one usually says that there is a quantum vortex with circulation $q$ somewhere within $\gamma$ when $q \neq 0$. As, however, $\gamma$ is arbitrary, one can conceiveably choose a path which will encompass more than vortex within the loop. It is therefore always tacitly assumed that this path $\gamma$ will encircle one and only one vortex in the fluid.

To describe the dynamical motion of these vortices, one usually appeals to classical fluid dynamics. Because the superfluid component of the fluid flow in three dimensions is nondissipative in nature, it behaves much like a classical ideal fluid. As ideal point vortices may also be present in the ideal fluid, one may then directly carry over and apply to quantum vortices in the superfluid the classical equations of vortex motion. Based on this argument, any motion of quantum vortices in the superfluid can be analyzed using classical fluid dynamics.

Let us now restrict ourselves to the case of two dimensions. Formally the above observations still hold, although we shall comment on certain problems with this argument latter on. Instead of having to work with vortex lines, we are now left with only point vortices, a very welcome simplification. In particular, by labeling the locations of the cores of the vortices by $\vec{x}^{\alpha}, \alpha=1, \ldots N$, the number of vortices in the fluid, we can treat the vortices as though they were point particles in and of themselves instead of being manifistations of a current flow in the film. Then by working in complex coordinants $z^{\alpha}=x^{\alpha}+i y^{\alpha}$, and $\bar{z}^{\alpha}=x^{\alpha}-i y^{\alpha}$, we appeal once again to fluid dynamics [5] and find that their lagrangian is 


$$
L=i \pi \hbar \rho_{0} \sum_{\alpha=1}^{N} q_{\alpha}\left(\frac{d z^{\alpha}}{d t} \bar{z}^{\alpha}-\frac{d \bar{z}^{\alpha}}{d t} z^{\alpha}\right)-K
$$

where

$$
K=-e_{k t} \sum_{q_{\alpha} \neq q_{\beta}}^{N} \log \left|\frac{z^{\alpha}-z^{\beta}}{a_{s}}\right|^{2},
$$

and $q_{\alpha}$ is the circulation of a vortex located at position $z^{\alpha}$ in the fluid. $a_{s}$ is some length scale for the system which is often identified as the size of the vortex core and $\rho_{0}$ is the average total density of the film while $e_{k t}$ is the KT energy scale: $e_{k t}=\rho_{0}(2 \pi \hbar)^{2} /(4 m)$. The infinite self-energy terms of the vortices have not been included in eq. (5). (One does not have to use fluid dynamics to obtain eq. (5). It can also be derived using the lagrangian for the microscopic field $\psi$ [15], [16].)

Now that we have a lagrangian for a "gas" of vortices in a two dimensional fluid, we can try to "quantize" this theory using naive canonical quantization. To do so, one takes as the generalized coordinate $\mathbf{q}^{\alpha}=z^{\alpha}$. Its canonical momentum is then $\mathbf{p}_{\alpha}=2 \pi i \hbar \rho_{0} q_{\alpha} z^{\alpha}$ and their Possion bracket is

$$
\left\{z^{\alpha}, \bar{z}^{\beta}\right\}=-i \delta_{\alpha \beta} /\left(2 \pi \hbar \rho_{0} q_{\alpha}\right)
$$

which makes perfectly good sense at a classical level. Next, because the lagrangian is linear in the velocities, we find that the classical hamiltonian $H_{c l}$ for the vortices is

$$
H_{c l}=\sum_{\alpha, \beta}^{N} \mathbf{p}_{\alpha} \frac{d \mathbf{q}_{\alpha}}{d t}-L=K
$$

and does not contain a kinetic piece but is instead all "potential".

If we now try to quantize this theory using naive canonical quantization, we immediately run into problems. First, the hamiltonian is all "potential" and is fully interacting. It does not have a "free" part about which we can perturb. Second, because the lagrangian is linear in the velocities, one may need to use Dirac's theory of contraints [17] while quantizing the theory to project out the unphysical states. Neither of these problems would seem to be insurmountable, however, until one tries to impliment canonical quantization; namely replacing the Poisson bracket by a commutator between two operators: $\left[z^{\alpha}, \bar{z}^{\beta}\right]=\delta_{\alpha \beta} /\left(2 \pi \rho_{0} q_{\alpha}\right)$. This, however, immediately implies that $z^{\alpha}$ does not commute with its complex conjugate 
$\bar{z}^{\alpha}$. Seemingly, the spatial coordinates of the quantum theory do not commute among themselves. Although technically this does not seem to present any great difficulties for two vortices [8], it does present certain conceptual problems and, fundamentally, is the reason why the vortex gas has yet to be successfully quantized. Moreover, from the work by [9] and [10], it is very doubtful that this naive quantization of the vortex gas can succeed.

Notice that the above arguments, expecially the form that $L$ takes, relies heavily on the analogy made between the classical ideal fluid and the superfluid. Actually, any and all quantum mechanical input into the subsequent arguments ends with the quantization condition eq. (3). As we have remarked, however, that although these arguments in support of the analogy hold quite well in three dimensions, the situation is not nearly as clear cut in two dimensions. This is because there are well known theorems which state that in two dimensions, $\langle\psi\rangle=0$ at all temperatures [12]- [13]. It is also known, however, that the KT transition, which is based on the presence of point vortices in the two dimensional superfluid, is a good model of the superfluid transition. Consequently, we shall assume that these vortices are present in the fluid and can be described at least heuristically in the same way that vortices in three dimensional fluids are.

\section{§3. The Single Vortex Hilbert Space}

We now begin the construction of the single vortex Hilbert space. As this construction is somewhat long and convoluted, we will first give an overview of how this is done. Fundamentally, what we shall be doing is determining explicitly the configuration of the fluid which will give rise to quantum vortices. First, by working with complex coordinates, a specific representation of the quantization condition eq. (3) is found in terms of functions on the complex plane. From it, the approximate form of the wavefunction describing a single vortex in the fluid with definite vorticity is obtained heuristically. A vorticity operator $(h / m) \mathcal{Q}$ is then defined which, when acting on a state of definite vorticity, returns simply its vorticity, an integer. Since this operator may be factorized into two pieces, we can define creation $c$ and annihilation operators $c^{\dagger}$ for the single vortex states. With the addition of the operator $c^{\dagger} c$, the algebra of relevant operators closes. Treating this algebra as fundamental and given, the single vortex Hilbert space is then constructed algebraicly in direct analogy with the SHO. 
We begin by finding the relevant operators using the quantization condition eq. (3). To do so, we recast the quantization condition

$$
\frac{h}{m} q=\int_{\gamma} \frac{j_{z}}{\rho} d z
$$

as a contour integral in the complex plane where

$$
j_{z}=\frac{\hbar}{2 m i}\left(\left\langle\psi^{\dagger}\right\rangle \frac{\partial\langle\psi\rangle}{\partial z}-\frac{\partial\left\langle\psi^{\dagger}\right\rangle}{\partial z}\langle\psi\rangle\right)
$$

In doing so, we are saying that $j_{z} / \rho$ is a meromorphic function while $j_{\bar{z}} / \rho$ gives no additional information and is redundent. Written in this form, we would expect heuristically that the wavefunction which describes a single vortex in the fluid with vorticity $q$ to have the form

$$
\phi \sim \rho^{1 / 2}\left(\frac{z}{\bar{z}}\right)^{q}
$$

where for convenience we now represent the wavefunctions of the fluid by $\phi$ instead of $\langle\psi\rangle$.

We then define the vorticity operator $(h / m) \mathcal{Q}$ as being that operator for which any wavefunction satisfying eq. (8) is an eigenstate of $\mathcal{Q}$. Namely, $\mathcal{Q}$ operating on a $\phi$ of the form given in eq. (10) gives: $\mathcal{Q} \phi=q \phi$. By definition, an eigenstate of this operator satisfies the quantization condition eq. (8) and visa versa. Using $\mathcal{Q}$ we may now directly impliment the quantization condition in terms of an operator acting on states of a Hilbert space.

With the form of $\phi$ given in eq. (10), we can choose a specific representation of $\mathcal{Q}$ in terms of differential operators

$$
\mathcal{Q}=\frac{1}{2}\left(z \frac{\partial}{\partial z}-\bar{z} \frac{\partial}{\partial \bar{z}}\right)
$$

as long as $\rho$ is a function of $|z|$ only. From this we would naively expect $\mathcal{Q}$ to be an hermitian operator, as required. Notice also that it is proportional to the generator of rotations in two dimensions. We next immediately see that it can be factorized

$$
\mathcal{Q}=\left[\frac{1}{\sqrt{2}} z \frac{\partial}{\partial \bar{z}}, \frac{1}{\sqrt{2}} \bar{z} \frac{\partial}{\partial z}\right]
$$

into a commutator of two other differential operators. With this observation it is then straightforward to construct the operators

$$
c \equiv \frac{1}{\sqrt{2}}\left(\frac{\bar{z}}{z}+\bar{z} \frac{\partial}{\partial z}\right) \quad, \quad c^{\dagger} \equiv \frac{1}{\sqrt{2}}\left(\frac{z}{\bar{z}}-z \frac{\partial}{\partial \bar{z}}\right),
$$


which, as we shall see, will serve as creation and annihilation operators for the vortex states. From their form we would expect $c$ and $c^{\dagger}$ to be adjoints of one another. Unfortunately, the situation is not nearly as clear cut, but for the sake of clarity we shall delay addressing this issue until $\S 4$. For now, we shall simply assume that they are truly adjoints of each other.

Defining $H_{c}=\epsilon c^{\dagger} c$, we find that $c, c^{\dagger}, H_{c}$ and $\mathcal{Q}$ have the following commutation relations:

$$
\begin{array}{rll}
{\left[c, c^{\dagger}\right]=I+\mathcal{Q} \quad,} & {\left[H_{c}, \mathcal{Q}\right]=0,} \\
{[c, \mathcal{Q}]=c \quad,} & {\left[H_{c}, c\right]=-\epsilon(I+\mathcal{Q}) c,} \\
{\left[c^{\dagger}, \mathcal{Q}\right]=-c^{\dagger} \quad,} & {\left[H_{c}, c^{\dagger}\right]=\epsilon c^{\dagger}(I+\mathcal{Q}) .}
\end{array}
$$

where $\epsilon$ is a constant having units of energy and shall be identified later. For reasons that will be made clearer in $\S \mathbf{4}$, we identify $H_{c}$ as the single vortex hamiltonian. For now, we simply note that $\mathcal{Q}$ commutes with $H_{c}$ and, consequently, is a conserved charge of the system. Notice also that its commutation relations with $c$ and $c^{\dagger}$ are precisely what one would expect for a charge operator.

From eq. (14) we see that the algebra $\mathcal{A}=\left\{I, c, c^{\dagger}, \mathcal{Q}, H_{c}\right\}$ closes. No other operators need to be introduced. We shall therefore take $\mathcal{A}$ as the basic set of physically relevant operators which are needed to describe a single vortex in a two dimensional superfluid. In addition, now that we have found the relevant operators for the single vortex system, we shall turn things around. Namely, we shall consider the abstract algebra eq. (14) as given and fundamental with $c^{\dagger}$ defined as the adjoint of $c$ and $\mathcal{Q}$ defined to be an hermitian operator. The explicit forms of these operators given in eqs. (11) and (13) are to be considered as specific representations of this algebra in terms of linear partial differential operators.

We do so because there are certain subtleties involved in finding faithful representation of eq. (14). Namely, apprearences notwithstanding, with the representation given in eq. (13) $c$ and $c^{\dagger}$ are not truly adjoints of one another. Although this problem can be resolved, doing so will take us somewhat afield. We shall therefore delay showing that a faithful representation of eq. (14) can be found until the next section and shall instead proceed with an algebraic construction of the single vortex Hilbert space based on this algebra.

Because $\mathcal{Q}$ and $H_{c}$ commute, one can find simultaneous eigenstates of both. We therefore provisionally define our single vortex Hilbert space $\mathcal{H}_{s}$ as being those states spaned by 
eigenstates of $\mathcal{Q}$ and $H_{c}$. Further constraints shall be put on $\mathcal{H}_{s}$ as needed. To charactorize the states of $\mathcal{H}_{s}$, we proceed in analogy with the SHO. Let $|\phi\rangle \in \mathcal{H}_{s}$ be an eigenstate of both $\mathcal{Q}$ and $H_{c}$ with eigenvalues $\lambda_{q}$ and $\lambda_{c}$ respectively. Then it is straightforward to show that for any positive integer $n$,

$$
\begin{aligned}
\mathcal{Q} c^{n}|\phi\rangle & =\left(\lambda_{q}-n\right) c^{n}|\phi\rangle, \\
\mathcal{Q} c^{\dagger^{n}}|\phi\rangle & =\left(\lambda_{q}+n\right) c^{\dagger^{n}}|\phi\rangle, \\
H_{c} c^{n}|\phi\rangle & =\left(\lambda_{c}+\frac{\epsilon}{2} n(n-1)-\epsilon n \lambda_{q}\right) c^{n}|\phi\rangle, \\
H_{c} c^{\dagger^{n}}|\phi\rangle & =\left(\lambda_{c}+\frac{\epsilon}{2} n(n+1)+\epsilon n \lambda_{q}\right) c^{\dagger^{n}}|\phi\rangle .
\end{aligned}
$$

From this we note the following:

1. If $|\phi\rangle$ is an eigenstate of $\mathcal{Q}$ and $H_{c}$, then $c^{n}|\phi\rangle$ and $c^{\dagger^{n}}|\phi\rangle$ are also eigenstates of $\mathcal{Q}$ and $H_{c}$.

2. The operator $c\left(c^{\dagger}\right)$ operating on $|\phi\rangle$ will produce a state whose $\mathcal{Q}$-eigenvalue has been decreased (increased) by 1 . Since eigenstates of $\mathcal{Q}$ are states with definite vorticity, we can physically interpret $c$ as the annihilation operator for an unit of positive vorticity +1 while $c^{\dagger}$ is the creation operator for an unit of positive vorticity.

3. If $\lambda_{q}$ is an integer for any eigenstate of $\mathcal{Q}$, then all the eigenvalues of $\mathcal{Q}$ are integers.

4. Like the SHO, the eigenvalues of $H_{c}$ must be greater then zero. Unfortunately, this does not put as great a constraint on the spectrum of $H_{c}$ as it did for the SHO since the $H_{c^{-}}$-eigenvalues of both $c^{n}|\phi\rangle$ and $c^{\dagger^{n}}|\phi\rangle$ increases quadratically with $n$. No matter what the initial values of $\lambda_{c}$ and $\lambda_{q}$ are, as long as

$$
-\left(\lambda_{c}+1\right) \leq \lambda_{q} \leq \lambda_{c}
$$

for any one eigenstate of $H_{c}$ of the Hilbert space, all eigenstates of $H_{c}$ will have non-negative eigenvalues. One does not automatically obtain the quantization of the spectrum for $H_{c}$ as in the case of the SHO. We shall have to appeal to physical reasoning instead. 
Suppose that we are given a film which is completely at rest in the laboratory frame. Then there should be a state of the Hilbert space which represents the state of the fluid in which no vortices are present whatsoever. The fluid is completely quiescent. Let us denote this state by $|0\rangle$, which we shall call the ground state of the system. We require, on physical grounds, that it be present in $\mathcal{H}_{s}$. Then $\mathcal{Q}|0\rangle=0$ and from the above all the eigenvalues of $\mathcal{Q}$ for states in $\mathcal{H}_{s}$ are quantized.

Unfortunately, determining $\lambda_{q}$ does not put any bound whatsoever on $\lambda_{c}$ and we shall have to use further arguments. Since $|0\rangle$ represents the fluid at rest without any vortex excitations whatsoever, it must be the state of lowest energy. Any other state of the system containing vortices must have a higher energy than it does. Suppose, then, that $H_{c}|0\rangle=$ $\lambda_{0}|0\rangle$ where $\lambda_{0} \neq 0$. Then $c|0\rangle \neq 0$ and is instead a state with vorticity -1 from eq. (15). Moreover, we see that $H_{c} c|0\rangle=\lambda_{0}|0\rangle$. The state with vorticity -1 would thus have the same energy as the state with no vorticies whatsoever. As this is physically unreasonable, we shall require that $H_{c}|0\rangle=0$ also. Then from the definition of $H_{c}$, we find that $\left.|c| 0\right\rangle\left.\right|^{2}=0$ and $c|0\rangle=0 . \quad c$ annihilates the ground state and the only relevant states in $\mathcal{H}_{s}$ are linear combinations of $c^{\dagger^{n}}|0\rangle$.

With the presence of this ground state in $\mathcal{H}_{s}$, all the eigenvalues of $\mathcal{Q}$ and $H_{c}$ are quantized and can be enumerated by a single quantum number $n \geq 0$. The single vortex Hilbert space $\mathcal{H}_{s}$ is spanned by the states

$$
|n\rangle \equiv \frac{c^{\dagger^{n}}|0\rangle}{\sqrt{(n(n+1) / 2) !}},
$$

constructed from the ground state. From eq. (15) they have eigenvalues

$$
\mathcal{Q}|n\rangle=n|n\rangle \quad, \quad H_{c}|n\rangle=\frac{\epsilon}{2} n(n+1)|n\rangle .
$$

The vorticity of the state $|n\rangle$ is therefore $n h / m$, as expected.

We would therefore seem to be finished. Notice, however, that $\mathcal{H}_{s}$ contains only states of positive vorticity. Due to physical constraints on the energy of the ground state, all the negative vorticity states were removed from $\mathcal{H}_{s}$. This still leaves open the problem of the construction of the negative vorticity Hilbert space, however. Fortunately, doing so is straightforward. 
Let us define the operator $P$ such that

$$
P^{2}=1 \quad, \quad P \mathcal{Q} P=-\mathcal{Q}
$$

which is the parity operator as can be seen explicitly using the coordinate representation of $\mathcal{Q}$. We then define the operators

$$
d \equiv P c P \quad, \quad d^{\dagger} \equiv P c^{\dagger} P \quad, \quad H_{d} \equiv P H_{c} P
$$

and find that

$$
\begin{array}{rll}
{\left[d, d^{\dagger}\right]=I-\mathcal{Q} \quad,} & {\left[H_{d}, \mathcal{Q}\right]=0,} \\
{[d, \mathcal{Q}]=-d \quad,} & {\left[H_{d}, d\right]=-\epsilon(I-\mathcal{Q}) d,} \\
{\left[d^{\dagger}, \mathcal{Q}\right]=d^{\dagger} \quad, \quad\left[H_{d}, d^{\dagger}\right]=\epsilon d^{\dagger}(I-\mathcal{Q}) .}
\end{array}
$$

Proceeding just as before, we find that the only relevant states are of the form

$$
|m\rangle=\frac{d^{\dagger^{m}}|0\rangle}{\sqrt{(m(m+1) / 2) !}},
$$

where once again $|0\rangle$ is the ground state of the system. Moreover, $|m\rangle$ is an eigenstate of $\mathcal{Q}$ and $H_{d}$ with eigenvalues

$$
\mathcal{Q}|m\rangle=-m|m\rangle \quad, \quad H_{d}|m\rangle=\frac{\epsilon}{2} m(m+1)|m\rangle .
$$

The states $|m\rangle$ all have negative vorticity, but with the same dependence of the energy eigenvalues on $m$. We then define the Hilbert space $\overline{\mathcal{H}}_{s}$ as being spaned by simultaneous eigenstates of $\mathcal{Q}$ and $H_{d}$. Moreover, from eq. (23) we can physically interpret the operator $d$ $\left(d^{\dagger}\right)$ as the annihilation (creation) operator for an unit of negative vorticity. With the use of a parity operator $P$ we have thus mapped the algebra $\mathcal{A}$ acting on the single vortex Hilbert space $\mathcal{H}_{s}$, containing states of positive vorticity only, into $\overline{\mathcal{A}}=\left\{I, d, d^{\dagger}, \mathcal{Q}, H_{d}\right\}$ acting on $\overline{\mathcal{H}}_{s}$, containing only states with negative vorticity.

Let us now return to the question of the determination of the ground state of the Hilbert space. Remember that we required that there be a state in the Hilbert space, identified as the ground state, which is rotationally invariant. We shall now show that this is equivalent to there being a state of the Hilbert space which is parity invariant. Suppose that $|\phi\rangle \in \mathcal{H}$ such that $|\phi\rangle=P|\phi\rangle$. Moreover, suppose that $|\phi\rangle$ is an eigenstate of $\mathcal{Q}$, so that 


$$
\mathcal{Q}|\phi\rangle=\lambda_{q}|\phi\rangle .
$$

Then multiplying both sides with $P$

$$
P \mathcal{Q} P P|\phi\rangle=\lambda_{q} P|\phi\rangle .
$$

Since $P \mathcal{Q P}=-\mathcal{Q}$, we find that $\lambda_{q}=-\lambda_{q}$, and $\lambda_{q}=0$. Thus, the only eigenstate of $\mathcal{Q}$ which is also parity invariant has vanishing eigenvalue. Physically, our requirement that there exists a state of the Hilbert space which has zero $\mathcal{Q}$-eigenvalue is simply the requirement that there exists a state of the Hilbert space which is parity invariant. Moreover, this state may function as the ground state for both $\mathcal{H}_{s}$ and $\overline{\mathcal{H}}_{s}$.

\section{§4. Single Vortex State Wavefunctions}

The construction of the Hilbert space done in the previous section, while valid, was formal and algebraic in nature. In its construction we assumed that an inner product on the Hilbert space has already been defined and that a faithful representation of the algebra can be chosen. These assumptions will now be justified. Then, with the faithful representation of the algebra known, we shall explicitly construct the single vortex wavefunctions and physically interpret the results which were obtain abstractly in the previous section. As the negative vorticity states can be obtained from the positive vorticity states using the parity operator, we concern ourselves with only positive vorticity states in this section.

Since we originally started out with a specific choice of $c$ and $c^{\dagger}$ when constructing eq. (14), finding a faithful representation of this algebra would seem to be straightforward. One would only have to find the wavefunctions by solving a first order differential equation and then varify that the $c$ and $c^{\dagger}$ defined from eq. (13) are truly adjoints of each other. Unfortunately, appearances notwithstanding, they are not. If we try using the representation of $c$ and $c^{\dagger}$ given in eq. (13) to construct the wavefunctions, we would obtain wavefunctions which are singular at $|z|=0$. Due to this singularity they will not be normalizeable. If we try to correct for this by removing a disk of fixed radius from the origen and restricting the domain of the wavefunctions, the wavefunctions will become normalizeable, but we will then find that $c$ and $c^{\dagger}$ are no longer adjoints of each another. The removal of this disk generates a surface term when performing an integration by parts. As this surface term does not vanish, in this representation $c$ and $c^{\dagger}$ are not adjoints of one another and eq. (13) cannot be a faithful representation of the algebra. 
Curiously enough, $H_{c}$, defined though eq. (13), and the representation of $\mathcal{Q}$ in eq. (11) are hermitian operators even when the domain of the wavefunctions are restricted. To show that they are hermitian operators, two integrations by parts must be preformed, resulting in two surface terms which cancel one another. This suggests that the failure of eq. (13) to form a faithful representation of the algebra is not a fatal one and only slight modifications to eq. (13) only will be needed.

We begin by defining the type of wavefunctions we shall be constructing and their inner product on the single vortex Hilbert space $\mathcal{H}_{s}$. The domain of the wavefunctions will be the complex plane $\mathbf{C}$ with the inner product on the Hilbert space defined as

$$
\langle\phi \mid \mathcal{O} \phi\rangle \equiv \int_{\mathbf{C}} \phi^{\dagger} \mathcal{O} \phi d^{2} x,
$$

for any operator $\mathcal{O}$ on the Hilbert space and $\phi \in \mathcal{H}_{s}$. In addition, the wavefunctions are required to be $L^{2}$ integrable over $\mathbf{C}$, meaning that

$$
\int_{\mathbf{C}}|\phi|^{2} d^{2} x<\infty
$$

is finite for all $\phi \in \mathcal{H}$.

To find the correct representation of the algebra, we return to eq. (13) and consider its possible generalization by taking

$$
c=\frac{1}{\sqrt{2}}\left(\frac{\bar{z}}{z} g(z, \bar{z})+h(\bar{z}) \bar{z} \frac{\partial}{\partial z}\right) \quad, \quad c^{\dagger}=\frac{1}{\sqrt{2}}\left(\frac{z}{\bar{z}} \overline{g(z, \bar{z})}-\overline{h(\bar{z})} z \frac{\partial}{\partial \bar{z}}\right),
$$

where $g(z, \bar{z})$ is a function of both $z$ and $\bar{z}$ while for $c^{\dagger}$ to be the adjoint of $c, h(\bar{z})$ can be a function of $\bar{z}$ only. We now require that $c$ and $c^{\dagger}$ be a faithful representation of the algebra eq. (14). Taking once again eq. (11) as the representation of $\mathcal{Q}$, then $[c, \mathcal{Q}]=c$ requires that $\mathcal{Q} g=0$ and $h=0$. Since $h=h(\bar{z})$ only, $h$ must be a constant, which we already know is unity.

As $\mathcal{Q} g=0, g=g(|z|)$ only. Then from $\left[c, c^{\dagger}\right]$,

$$
1=\frac{1}{2}(g+\bar{g})+\frac{1}{2} r \frac{d}{d r}\left(\frac{g+\bar{g}}{2}\right),
$$

where $r=|z|$. Since all other commutation relations follow from these two, no additional equations for $g$ are found. In particular, notice that there is no constraint on the imaginary 
part of $g$. For convenience, we shall choose $g$ to be real, and shall remark on other choices later. Then

$$
g=1-\frac{a^{2}}{2 r^{2}},
$$

where $a$ is some constant yet to be determined. Notice that when $a=0, g=1$ and we are back to eq. (13).

Let $\phi_{0}$ be the ground state. Then by definition $c \phi_{0}=0$, and

$$
z \frac{\partial \phi_{0}}{\partial z}+g \phi_{0}=0 .
$$

Because the ground state is rotationally invariant, solving eq. (31) gives

$$
\phi_{0}=\frac{a}{\sqrt{\pi}} \frac{e^{-a^{2} /\left(2 r^{2}\right)}}{r^{2}},
$$

with the correct normalization. Notice that when $g=1, \phi_{0}$ has the singularity at $r=0$ we mentioned at the beginning of this section. Other eigenstates $\phi_{n}$ of $\mathcal{Q}$ can be found by successive application of $c^{\dagger}$ on $\phi_{0}$. For the first few $n$,

$$
\begin{aligned}
\phi_{1} & =\sqrt{2}\left(\frac{z}{\bar{z}}\right) g \phi_{0}, \\
\phi_{2} & =\frac{1}{\sqrt{3}}\left(\frac{z}{\bar{z}}\right)^{2}\left(2 g^{2}+2 g-1\right) \phi_{0}, \\
\phi_{3} & =\frac{2}{3}\left(\frac{z}{\bar{z}}\right)^{3}\left(g^{3}+3 g^{2}-1\right) \phi_{0} .
\end{aligned}
$$

where we have normalized all these states to unity. We therefore expect that in general

$$
\phi_{n}=\left(\frac{z}{\bar{z}}\right)^{n} G_{n}(g) \phi_{0},
$$

where $G_{n}(g)$ is a polynomial in $g$. Using eq. (28), we see that it satisfies the recursion relation:

$$
\begin{aligned}
G_{n}(g) & =-\frac{1}{\sqrt{2}} \frac{e^{-2 g}}{(1-g)^{n}} \frac{d}{d g}\left[e^{2 g}(1-g)^{n+1} G_{n-1}\right], \\
& =\left(\frac{-1}{\sqrt{2}}\right)^{n} \frac{e^{-2 g}}{(1-g)^{n}}\left\{\frac{d}{d g}(1-g)^{2}\right\}^{n} e^{2 g},
\end{aligned}
$$

given $G_{0}=1$. Notice also that $\phi_{n}$ has precisely the form we expected from the heuristic arguments given in $\S 3$. 
$\phi_{n}$ are functions which are defined throughout C. In particular:

$$
\lim _{|z| \rightarrow 0}\left|\phi_{n}(z, \bar{z})\right|^{2}=0 \quad, \quad \lim _{|z| \rightarrow \infty}\left|\phi_{n}(z, \bar{z})\right|^{2}=0,
$$

with the presence of $g$ in eq. (28) serving as a short distance regulator. We no longer have any singularity problems and it is trivial to show that not only are the $c$ and $c^{\dagger}$ given in eq. (28) adjoints of one another, but also that the representation of $\mathcal{Q}$ given in eq. (11) truly corresponds to an hermitian operator. We have therefore found a faithful representation of the algebra and the formal analysis done in $\S 3$ is now justified.

In retrospect, the problem that we had with eq. (13) could have been anticipated. Using a coordinate represetation of the algebra, eigenstates of $\mathcal{Q}$ are proportional to $e^{2 i \theta}$, where $\theta$ is the phase of $z$. Yet, this phase is not well defined at $r=0$, which is precisely where the singularity of the wavefunctions constructed from eq. (13) occurs. This singularity can be seen explicitly by setting $g=1$ in eq. (32). A cutoff must be introduce at small $r$, which is essentially the role that $a$ plays. Also, notice that eq. (13), unlike the creation and annihilation operators for the SHO, does not contain within themselves a length scale. Yet the wavefunction must have units of inverse length. A length scale must be therefore be introduced, which is a role that $a$ also serves.

With the correct representation of the the algebra known, and the wavefunction of the single vortex states constructed, we can now return to our premise that the algebra eq. (14) is the correct quantum mechanical description of a quantum vortices in a two dimensional superfluid. Specifically, the question is whether or not the algebra eq. (14), and the wavefunctions construced from it, actually does describe the single vortex state in the superfluid. As we have seen, $\mathcal{Q}$ already has all the agreeable properties we would expect from a vorticity operator. Moreover, the wavefunctions have precisely the form we expected from heuristic arguments. The question is whether or not $H_{c}$ does. One way to answer this question is to compare the features $H_{c}$ with some of the properties of quantum vortices known from classical fluid dynamics. By doing so we shall also be able to identify physically the two constants in the theory: $\epsilon$ and $a$. We begin with the eigenstates of $H_{c}$.

There are two features of the classical hamiltonian eq. (5) which are essential in deriving the renormalization group equations for the KT phase transition. First, the classical energies are proportional to $q^{2}$, the vorticity of the vortex. Second, because of the logarithmic 
interaction, under scaling $z \rightarrow \chi z, K \rightarrow K-2 e_{k t} \sum_{\alpha \neq \beta}^{N} q_{\alpha} q_{\beta} \log \left|\chi / a_{s}\right|$ and gets shifted by a constant. Since the hamiltonian is defined only up to a constant anyway, it is effectively invariant under scaling.

For $H_{c}$ to be the correct vortex hamitonian, it must have at least these two features in common with the classical hamiltonian. From eq. (18) we see that the eigenvalues of $H_{c}$ is proportional to $n(n+1)$. As expected, for large $n$ any differences between this dependence and $n^{2}$ is negligible. As for the scaling, let us write $H_{c}$ in terms of differential operators,

$$
\begin{aligned}
H_{c} & \equiv \epsilon c^{\dagger} c \\
& =-\frac{\epsilon}{2}\left\{|z|^{2} \frac{\partial^{2}}{\partial z \partial \bar{z}}+g \bar{z} \frac{\partial}{\partial \bar{z}}+(1-g) z \frac{\partial}{\partial z}+\left(1-g^{2}\right),\right\}
\end{aligned}
$$

and consider the limit where $r \gg a$. In this limit, $g \rightarrow 1$ and we can see that $H_{c}$ is also invariant under scaling $z \rightarrow \chi z$. But only in this limit. This is to be expected, however. The classical hamiltonian has a logarithmic singularity when $z^{\alpha} \rightarrow z^{\beta}$, and for it to be well defined, the interaction must be regularized. Any regularization scheme will ruin the scaling behavior at small $r$, but at large $r$ the classical hamiltonian is approximatedly scale invariant, which is precisely the properties that $H_{c}$ has.

As $H_{c}$, and its eigenvalues, have many of the same properties as the classical hamiltonian, we were justified in identifying it as the single vortex hamiltonian. The unknown energy scale $\epsilon$ can now be identified as $\epsilon_{k t}$. Each of the eigenstates of $H_{c}$ thus correspond to the total energy of a single vortex in the fluid with vorticity $n$. Classically, we would call this the "self-energy" of the vortex, as this is the amount of energy needed to creat a vortex in the fluid. Unlike the classical self-energy, which is infinite, all eignvalues of $H_{c}$ are finite, however.

Mathematically, $a$ in eq. (32) serves as an short distance regulator. With its presence the wavefunction vanishes at $r=0$ and there are no problems with the definition of $\theta$. It is, however, at this point questionable as to whether or not $a$ has any other physical relevance since its value does not affect the eigenvalues of $H_{c}$. To gain some physical insight into what other role it may play, consider the usual quantum mechanical hamiltonian

$$
H_{L}=-\frac{\hbar^{2}}{2 m} \nabla^{2}=-\frac{2 \hbar^{2}}{m} \frac{\partial^{2}}{\partial z \partial \bar{z}} .
$$

The actual hamiltonian for the vortices is $H_{c}$, of course, not $H_{L}$. We consider $H_{L}$ only to 
make a connection with the usual calculation of the average energy of a vortex. In doing so we find that

$$
\left\langle\phi_{0} \mid H_{L} \phi_{0}\right\rangle=\frac{\hbar^{2}}{m a^{2}},
$$

and, in terms of $H_{L}$, we would say that $\phi_{0}$ is not a state with zero energy. Correcting this would only involve shifting $H_{L}$ by a constant, however. Of more interest is

$$
\begin{aligned}
\left\langle\phi_{1} \mid H_{L} \phi_{1}\right\rangle & =6 \frac{\hbar^{2}}{m a^{2}}, \\
\left\langle\phi_{2} \mid H_{L} \phi_{2}\right\rangle & =26 \frac{\hbar^{2}}{m a^{2}},
\end{aligned}
$$

and we find that $\left\langle\phi_{2} \mid H_{L} \phi_{2}\right\rangle \approx(2)^{2}\left\langle\phi_{1} \mid H_{L} \phi_{1}\right\rangle$. Once again the energies have the correct dependence on charge. Comparing eq. (40) with $K$, we therefore identify $a^{2}=1 / \rho_{0}$.

Let us now try to calculate $\Delta x \equiv \sqrt{\left\langle|z|^{2}\right\rangle-|\langle z\rangle|^{2}}$, the rms "position" of the vortex. By symmetry, $\langle z\rangle=0$. However,

$$
\left\langle|z|^{2}\right\rangle \equiv \int_{\mathbf{C}}|z|^{2}|\phi|^{2} d^{2} x \sim a^{2} \log R / a
$$

where $R$ is the large distance cutoff and represents the "size" of the system. Consequently, $\left\langle|z|^{2}\right\rangle$ diverges logarithmically and we cannot say that the vortex is localized at any one point in the fluid.

The parameter $a=1 / \sqrt{\rho}_{0}$ is offen refered to in the literature as the "core size" of a vortex. Although we see that the classical notion that the vortex is localized at any specific point in the fluid is incorrect, let us see if we can still nevertheless consider $a$ as an effective size of the vortex. Consider the function $\left|\phi_{n}\right|^{2}$, which is rotationally symmetric. From eq. (36) we know that all $\left|\phi_{n}\right|^{2}$ have a zero at $r=0$. Although $\left|\phi_{0}\right|^{2}$ has no other zeros, other $\left|\phi_{n}\right|^{2}$ will. In fact, $\left|\phi_{1}\right|^{2}$ has an additional zero at $r=a / \sqrt{2} ;\left|\phi_{2}\right|^{2}$ has two other zeros at $r=a /(3 \pm \sqrt{3})$; while $\left|\phi_{3}\right|^{2}$ has three aditional zeros at $r=a /(2.78), r=a /(1.82)$ and $r=a /(0.967)$. Since $\left|\phi_{n}\right|^{2}$ cannot be negative, there are corresponding local maxima of $\left|\phi_{n}\right|^{2}$ at points in between two zeros. Due to the $1 / r^{4}$ behavior of $\left|\phi_{n}\right|^{2}$, we would expect the largest maxima to be between $r=0$ and the next nearest zero of the function. For $n=1,2,3$, they all fall within $r=a / \sqrt{2}$. Because the probablity density is highest for $r \leq a / \sqrt{2}$, we may identify $a / \sqrt{2}$ as the effective "core size" of a vortex. Indeed, with this 
identification the independence of the eigenvalues of $H_{c}$ on its value can be understood. It is once again a reflection of the classical hamiltonian. If we scale $a_{s} \rightarrow l a_{s}$, the classical hamiltonian $K$ gets shifted only by an irrelevant constant. The classical hamiltonian, like $H_{c}$, is also effectively independent of the "size" of the vortex core.

The interpretation of $a$ as a core size runs into problems, however, if we now reconsider the arbitrariness in the choice of $g$. Remember that we can add to $g$ a function $g_{I}$ of $|z|$ which is purely imaginary, $\overline{g_{I}}=-g_{I}$, and $c$ and $c^{\dagger}$ will still be faithful representations of the vortex algebra. From eq. (34) we see that doing so will alter the ground state wavefunction $\phi_{0}$ by the multiplication of a complex phase $e^{i \xi}$ where

$$
g_{I}=-\frac{i}{2} r \frac{d \xi}{d r}
$$

and $g_{I}$ is pure imaginary. Therefore, a shift in $g$ is equivalent to a local gauge transformation of $\phi_{0}$. Choosing $g$ to be real corresponds to a choice of guage. This clearly will not change the convergence properties of $\phi_{0}$, or any of the other $\phi_{n}$, as long as $\left|g_{I}\right|$ does not diverge faster

than $e^{-a^{2} / r^{2}}$. $\phi_{n}$ will still, of course, be eigenstates of $H$ and $\mathcal{Q}$ with the same eigenvalues, since they do not depend on $g .\left|\phi_{n}\right|^{2}$ does, however, and the probability density changes with different $g$. Notice also that changing $g$ will result in changing $a$, what we have identify as the "core size". Consequently, the concept of a definite size of the vortex is therefore not well defined as it depends on the choice of $g$. We shall remark further on this later in $\S 7$.

\section{$\S 7$. Conclusion}

By using the observation that we already know which states of the fluid are of importance, it was straightforward to develope a quantum mechanical description of vortices in the fluid. What we ultimately obtained, however, is a single vortex hamiltonian which is very much different than what one normally encounters. As the classical equations of vortex motion, Kirchoff's equations, are themselves drastically different from the usual equations of motion of Newtonian mechanics, this should not be too surprizing. What is of more importance and relevance is that $H_{c}$, and its eigenvalues, has many of the same properties as the classical hamiltonian. Namely, that $H_{c}$ is scale invariant at large $r$ while its eigenvalues are proportional to the square of the vortex charge, and is independent of the "core size" of the vortex. For these reasons we believe that $H_{c}$, and its eigenstates, form an accurate quantum mechanical description of the single vortex system. 
The question then becomes how such an hamiltonian can come about from the usual microscopic ${ }^{4} \mathrm{He}$ hamiltonian. As ours is essentially a phenomenological description of the single vortex system, this cannot be answered within this formalism. $H_{c}$ must instead be seen as an effective hamiltonian of the fluid, albeit one which differs drastically from the ones we are accustomed to using. As for how it can araise, we can only note that at very low temperatures ${ }^{4} \mathrm{He}$ is strongly interacting. $H_{c}$ may thus araise as an effective hamiltonian of this strongly interacting theory, especially since vortices are the result of a collective property of the system. Quantum mechanically, they represent a definite excitation state of the quantum fluid (what is sometimes refered to as a psuedo-particle), while classically they are a specific configuration of the current flow in an ideal fluid.

We have been calling $\phi_{n}$ the single vortex wavefunctions. This term is a somewhat vague, however, and was mainly used as a matter of convenience. Physically, we should instead interpret each $\phi_{n}$ as representing an excitation of the superfluid which give rise to a single vortex with vorticity $n$ in the fluid. Its norm, $\left|\phi_{n}\right|^{2}$, is therefore the number density for the ${ }^{4} \mathrm{He}$ atoms in this state. (The integral of $\left|\phi_{n}\right|^{2}$ is then the total number $N_{v}$ of ${ }^{4} \mathrm{He}$ atoms in this state. Although we have normalized $\phi_{n}$ to unity, we could have just as well normalized it to $N_{v}$.) It would therefore seem that from the results of $\S 4$ we have uniquely determined the density of the fluid needed to creat a quantum vortex with vorticity $n$ in the fluid. Notice, however, that $\phi_{n}$ is not unique. It is dependent on $g$, which itself is defined only up to an arbitrary function of $|z|$. Different choices of $g$ will result in different $\phi_{n}$, although $\left|\phi_{0}\right|^{2}$ is always uniquely defined. Consequently, as the eigenvalues of $H_{c}$ and $\mathcal{Q}$ do not depend upon $g$, there are many different excitations of the fluid which will give raise to the same vorticity state. The condition that the superfluid is in a state of definite vorticity is not sufficient to determine the state of the fluid uniquely.

Notice that in general $\left|\phi_{n}\right|$ is not a constant thoughout the fluid. In the usual theory of vorticies in the superfluid, however, the density of the fluid was necessarily a constant almost everywhere. (Only when one gets close to the vortex core does the density vary.) And it was only in this manner that the analogy between the superfluid and the classical ideal fluid could be used. We now see that this condition is unnecessarily restrictive. Due to the freedom in choosing $g$, there are infinitely many different excitations of the fluid, not 
only the one with constant density, which have the same vorticity and, more importantly, the same energy.

In this paper we have only consider the behavior of a single vortex in the fluid. The next step is to develope a many vortex formulation of this system [19. Since we have annihilation and creation operators for these vortices, this is straightforwardly done in complete analogy to the case of the SHO. Once this is accomplished the statistical mechanics of the many vortex system can be studied within an algebraic formalism and compared with the results obtained using the KT analysis of the classical vortex gas.

\section{Acknowledgements}

This work was supported in part by the R.O.C. NSC Grant Nos. NSC84-2112-M-001-022. 


\section{REFERENCES}

1 J. M. Kosterlitz and J. D. Thouless, J. Phys. C6 1181 (1973).

2 J. M. Kosterlitz, J. Phys. C7 1046 (1974).

3 D. R. Nelson and J. M. Kosterlitz, Phys. Rev. Lett. 391201 (1977).

4 A. D. Speliotopoulos, and H. L. Morrison, J. Phys. A24 5029 (1991).

$5 \quad$ K. O. Friedrichs, Special Topics in Fluid Dynamics, (Gordon and Breach, New York, 1966), Chapter 19.

$6 \quad$ D. J. Bishop and J. D. Reppy, Phys. Rev. Lett. 401727 (1978).

$7 \quad$ L. Onsager, Nuovo Cimento Suppl. 6249 (1949).

$8 \quad$ J. L. McCauley, J. Phys. A12 1999 (1979).

$9 \quad$ G. A. Goldin, R. Menikoff, and D. H. Sharp, Phys. Rev. Lett. 512246 (1983).

10 G. A. Goldin, R. Menikoff, and D. H. Sharp, Phys. Rev. Lett. 582162 (1987).

11 D. R. Tilley and J. Tilley, Superfluidity and Superconductivity, third ed., (Adam Hilger, Bristol, 1990), Chapters 2, 6.

12 P. C. Hohenberg, Phys. Rev. 158383 (1967).

13 N. D. Mermin and H. Wagner, Phys. Rev. Lett. 171133 (1966).

14 J. C. Garrison, J. Wong, and H. L. Morrison, J. Math. Phys. 131735 (1972).

15 A. D. Speliotopoulos and H. L. Morrison, Phys. Lett. A141 284 (1989). H. L. Morrison, U. K. Albertin, and J. V. Lindesay, Phys. Lett. A108 8 (1985).

16 A. D. Speliotopoulos and H. L. Morrison, J. Phys, A26 3527 (1993).

17 P. A. M. Dirac, Canadian J. Math., 2 (1950) 129.

18 P. Minnhagen and G. G. Warren, Phys. Rev., B24 (1981) 2526.

19 A. D. Speliotopoulos, in preparation. 